\title{
MicroRNA-342-3p suppresses proliferation and invasion of nasopharyngeal carcinoma cells by directly targeting $\mathrm{Cdc42}$
}

\author{
LU SHI $^{*}$, RUOWEN XIAO $^{1 *}$, MENGHE WANG $^{2 *}$, MEIYIN ZHANG $^{1}$, NUOQING WENG $^{1}$, \\ XINGE ZHAO $^{1}$, X.F. STEVEN ZHENG ${ }^{1,3}$, HUIYUN WANG ${ }^{1}$ and SHIJUAN MAI ${ }^{1}$
}

\begin{abstract}
${ }^{1}$ State Key Laboratory of Oncology in South China, Collaborative Innovation Center for Cancer Medicine, Guangdong Key Laboratory of Nasopharyngeal Carcinoma Diagnosis and Therapy, Sun Yat-sen University Cancer Center, Guangzhou, Guangdong 510060; ${ }^{2}$ Department of Endocrinology, People's Hospital of Xinjiang Uyghur Autonomous Region, Urumqi, Xinjiang 830063, P.R. China; ${ }^{3}$ Rutgers Cancer Institute of New Jersey, Rutgers University,
\end{abstract} New Brunswick, NJ 08901, USA

Received April 13, 2018; Accepted July 31, 2018

DOI: $10.3892 / o r .2018 .6642$

\begin{abstract}
Deregulated microRNAs play an important role in the development and progression of various types of cancer. In our previous study, we observed that microRNA-342-3p (miR-342-3p) was one of the most markedly downregulated microRNAs in two nasopharyngeal carcinoma (NPC) cell lines compared to non-neoplastic cells by using whole genome small RNA sequencing. In the present study, we confirmed that the expression of miR-342-3p was significantly reduced in NPC tissues compared with normal nasopharyngeal epithelial tissues. Overexpression of miR-342-3p inhibited proliferation, epithelial-mesenchymal transition (EMT), migration and invasiveness of NPC cells. In addition, we observed that Cdc42, a Rho GTPase family member involved in cell proliferation and metastasis, is a direct target of miR-342-3p. Additionally, ML141, a small-molecule inhibitor of Cdc42, efficiently suppressed the invasion of NPC cells compared with the control cells. Finally, we analyzed NPC tissues derived from 10 NPC patients and subjected them to quantitative RT-PCR and immunohistochemistry assays for concomitant determination of the expression levels of miR-342-3p and Cdc42. Our results revealed that miR-342-3p levels were significantly inversely correlated with the protein levels of its target Cdc 42 . The results of the present study indicated that miR-342-3p
\end{abstract}

Correspondence to: Professor Shijuan Mai or Professor Huiyun Wang, State Key Laboratory of Oncology in South China, Collaborative Innovation Center for Cancer Medicine, Guangdong Key Laboratory of Nasopharyngeal Carcinoma Diagnosis and Therapy, Sun Yat-sen University Cancer Center, 651 East Dongfeng Road, Guangzhou, Guangdong 510060, P.R. China

E-mail: maishj@sysucc.org.cn

E-mail:wanghy@mail.sysu.edu.cn

*Contributed equally

Key words: miR-342-3p, proliferation, invasion, NPC, Cdc42 inhibited NPC tumor growth and invasion by directly targeting the $\mathrm{Cdc} 42$ pathway

\section{Introduction}

Nasopharyngeal carcinoma (NPC) is a type of cancer arising from the nasopharynx epithelium and has a high prevalence in south Asia and Africa. Its etiological agent includes Epstein-Barr virus (EBV) infection, environmental and diet factors and genetic susceptibility (1). Due to its insidious location and lack of early symptoms, NPC patients tend to present with a late-stage diagnosis. Although NPC is radiosensitive, the patient 5-year survival rate remains low, largely due to regional lymph node and distant metastasis and locoregional recurrence. The outcomes of advanced-stage NPC are markedly worse compared with those in earlier stages (2). Thus, identifying specific biomarkers with diagnostic value and understanding the molecular mechanisms that regulate the invasion and metastasis of NPC is urgently needed.

MicroRNAs (miRNAs) are small non-coding singlestranded RNAs of 19-25 nucleotides in length, which regulate $\sim 60 \%$ of gene expression by binding to the 3 ' untranslated region (UTR) of the mRNA targets through the seed sequence (3). A growing body of literature indicates that miRNAs play important roles in most biological processes, such as development, proliferation, differentiation, cell cycle control and cell death, tumorigenesis and adaptation to stress (4). Thus, miRNAs have emerged as potential biomarkers or therapeutic targets for human diseases including cancer.

To date, aberrant miRNA expression has been reported in the development and progression of NPC. For example, miR-124 (5), miR-125a-5p (6) and miR-101 (7) acted as tumor suppressors by inhibiting NPC cell growth, or by promoting cell apoptosis. miR-200a and miR-200b suppressed NPC cell migration and invasion by targeting ZEB2, CTNNB1 and Notch1 $(8,9)$. miR-139-5p, miR-3188 and miR-29c were reported to enhance the sensitivity of NPC to chemotherapy and radiotherapy (10-12). Conversely, some miRNAs can act as oncogenes in NPC. Upregulation of miR-19a and miR-222 
promoted NPC cell proliferation by directly targeting transforming growth factor $\beta$ receptor 2 or PTEN $(13,14)$.

In our previous study, whole-genome small RNA deep sequencing was performed on NPC cell lines C666-1, CNE2 and the non-neoplastic cell line NP69, to characterize miRNA expression profiles in NPC cells (unpublished data). The aberrantly expressed miRNAs were subsequently validated by real-time quantitative PCR in NPC and non-cancerous nasopharyngitis biopsies. Of these differentially expressive miRNAs, miR-342-3p was identified to be significantly downregulated in NPC cell lines and tissues. However, thus far, the expression profile of miR-342-3p and its role in NPC have not been characterized.

In the present study, we investigated the tumor-suppressive roles of miR-342-3p in NPC cells. We revealed that overexpression of miR-342-3p inhibited NPC cell proliferation and invasion by directly targeting $\mathrm{Cdc} 42$.

\section{Materials and methods}

Patients and samples. Twenty-two NPC tissue samples and 15 nasopharyngitis tissues were collected and total RNA was extracted for further RT-qPCR assay. Formalin-fixed, paraffin-embedded tissues (FFPTs) of 10 NPC tissues were acquired from NPC patients who were firstly diagnosed with NPC at the Sun Yat-sen University Cancer Center (SYSUCC; Guangzhou, China) from January 2007 to December 2007. The patients were histologically and clinically diagnosed with NPC and assessed according to the TNM staging of The International Union against Cancer. None of the patients were subjected to radiotherapy or chemotherapy prior to biopsy sampling. The present study was approved by the Research Ethics Committee of SYSUCC, and written informed consent was obtained from all patients.

Immunohistochemical staining. Paraffin sections were prepared from NPC patients and immunohistochemistry (IHC) assays were performed according to the manufacturer's instructions to detect the protein expression. The antibody rabbit anti-Cdc42 (1:200; cat. no. 10155-AP-1) was purchased from Proteintech Group Inc., (Wuhan, China). In brief, slides with paraffin sections underwent deparaffinage and aquation. Then $3 \%$ hydrogen peroxide was used to block endogenous peroxidase activity and antigen retrieval was performed by boiling slides in ethylene diamine tetraacetic acid (EDTA) (1 mmol/1, pH 8.0). The slides were incubated with the primary antibody anti-Cdc 42 overnight at $4^{\circ} \mathrm{C}$. The staining score was assessed by two pathologists independently. The intensities were graded as 0 (negative), 1 (weakly positive), 2 (moderately positive) and 3 (strongly positive). The abundance of positive cells was graded from 0 to $100 \%$. The staining score was determined using the following formula: Overall scores $=$ percentage score $\mathrm{x}$ intensity score $\mathrm{x} 100$.

NPC cell culture and transfection. The human NPC cell lines C666-1, SUNE1, 6-10B and 5-8F used in the present study, were cultured in RPMI-1640 medium (Gibco; Thermo Fisher Scientific, Inc., Waltham, MA, USA) with $10 \%$ fetal bovine serum (FBS; Invitrogen Life Technologies; Thermo Fisher Scientific, Inc.). The human immortalized nasopharyngeal epithelial cell line, NP69, was grown in defined-keratinocyte serum-free medium (KSFM) supplemented with EGF (Invitrogen Life Technologies; Thermo Fisher Scientific, Inc.). The aforementioned cells were cultured in a humified atmosphere with $5 \% \mathrm{CO}_{2}$ at $37^{\circ} \mathrm{C}$. All NPC cell lines and NP69 cell were provided generously by Professor Musheng Zeng (Sun Yat-sen University Cancer Center, Guangzhou, China). All cells were negatively tested for mycoplasma contamination before use, and authenticated based on STR fingerprinting before use at The Medicine Lab of Forensic Medicine Department of Sun Yat-sen University. miR-342-3p mimics were synthesized by Suzhou GenePharma Co., Ltd. (Suzhou, China) as follows: miR-342-3p sense, 5'-UCUCACACAGAA AUCGCACCCGU-3' and antisense, 5'-GGGUGCGAUUUC UGUGUGAGAUU-3'). miR-342-3p mimics were transfected into 6-10B cells at a final concentration of $15 \mathrm{nM}$ using Lipofectamine 3000 (Invitrogen Life Technologies; Thermo Fisher Scientific, Inc.).

Reverse quantitative polymerase chain reaction ( $R T-q P C R)$. Total RNA was extracted from tissues or cells with TRIzol reagent (Invitrogen Life Technologies; Thermo Fisher Scientific, Inc.) following the manufacturer's protocol. cDNA was obtained using GoScript Reverse Transcription Mix (Promega Corp., Madison, WI, USA) and Bulge-Loop ${ }^{\mathrm{TM}}$ miRNA qPCR Primer set for miR-342-3p (Guangzhou RiboBio Co., Ltd., Guangzhou, China). qPCR was performed with GoTaq $^{\circledR}$ qPCR Master Mix (Promega Corp.). The standard cycling program was performed as follows: hot-start activation: $95^{\circ} \mathrm{C}$ for $2 \mathrm{~min}, 1$ cycle; denaturation: $95^{\circ} \mathrm{C}$ for $15 \mathrm{sec}$, 40 cycles; annealing/extension: $60^{\circ} \mathrm{C}$ for $60 \mathrm{sec}$; dissociation: $75^{\circ} \mathrm{C}, 1$ cycle. The mRNA expression level was examined by the $2^{-\Delta \Delta \mathrm{Cq}}$ method (15) and normalized by internal control U6. The sequence of Cdc42 primer for RT-qPCR assay was as follows: Forward, 5'-CCATCGGAATATGTACCGACTG-3' and reverse, 5'-CTCAGCGGTCGTAATCTGTCA-3'.

Cell proliferation and colony formation assay. Cell growth was determined by Cell Counting Kit-8 (CCK-8; Dojindo Molecular Technologies, Inc., Kumamoto, Japan). In brief, 1,000 cells/well were suspended into a 96-well-plate and pre-incubated for $24 \mathrm{~h}$ in a humidified incubator $\left(5 \% \mathrm{CO}_{2}\right.$ at $\left.37^{\circ} \mathrm{C}\right)$. Following the addition of $10 \mu \mathrm{l} \mathrm{CCK}-8$ solution to each well of the plate and incubation of the plate for $3 \mathrm{~h}$, the absorbance at $450 \mathrm{~nm}$ was determined using a microplate reader (Bio-Rad Laboratories, Inc., Hercules, CA, USA). All experiments were performed in triplicates.

Concerning the colony formation assay, 6-10B miR-342-3p mimics, as well as their control cells were seeded into a 6-well-plate (500 cells/well) with complete medium. The cells were grown for 12 days at $37^{\circ} \mathrm{C}$ with $5 \% \mathrm{CO}_{2}$. To visualize the colony formation, the cells were fixed with methanol and stained with $0.1 \%$ crystal violet (Beyotime Institute of Biotechnology, Shanghai, China) and observed under an inverted fluorescent microscope. The number of colonies $>0.1 \mathrm{~mm}$ diameter was counted. The colony formation of each cell line was performed in triplicate.

Luciferase reporter assay. The wild-type (wt) or mutant (mt) Cdc42-3'UTR luciferase reporter vector was designed on the 
Table I. miR-342-3p is downregulated in nasopharyngeal carcinoma C666-1 cells compared with NP69 cells in microRNA-Seq results.

\begin{tabular}{lllcr}
\hline & \multicolumn{2}{c}{ Mean expression value } & & \\
\cline { 2 - 3 } MicroRNA & NP69 & C666-1 & $\log 2$ (fold change) & P-value \\
\hline hsa-miR-342-3p & 327.16 & 26.36 & $-3.64($ C666-1 vs. NP69) & 0.006960605 \\
\hline
\end{tabular}

basis of miR-342-3p binding sites. In brief,4x104293T cells/well were seeded into a 24-well plate. Following incubation for $24 \mathrm{~h}$ at $37^{\circ} \mathrm{C}$ with $5 \% \mathrm{CO}_{2}$, using Lipofectamine 3000 reagent (Invitrogen Life Technologies; Thermo Fisher Scientific, Inc.), $1,000 \mathrm{ng}$ firefly luciferase reporter plasmid was co-transfected with 10 ng pRL-TK Renilla plasmid into cells, combined with miR-342-3p mimics or negative control. In accordance with the manufacturer's instructions, the luciferase and Renilla signals were determined using the Dual-Luciferase Reporter Assay kit (Promega Corp.). The experiments were repeated independently three times.

Wound healing and invasion assay. A scratch wound healing assay was performed to assess the cell migration rate. Cells transfected with miR-342-3p mimics were cultured in a 6-well plate. Subsequently, as the cells reached sub-confluence, with a sterile micropipette tip, a scratching wound was generated and images were captured under an inverted fluorescent microscope at 0 and $48 \mathrm{~h}$. The culture medium was replaced by serum-free RPMI-1640 medium. The distance between either side of the scratch in images captured by the inverted fluorescent microscope was assessed to evaluate the cell migration rate.

Cell invasion assay was conducted using cell culture inserts with 8- $\mu \mathrm{m}$ PET track-etched membranes (Falcon; BD Biosciences, Franklin Lakes, NJ, USA). Tumor cells were placed into the upper chambers $\left(5 \times 10^{4}\right.$ cells/well $)$ with $200 \mu \mathrm{l}$ serum-free medium, whereas the lower chambers in the 24-well-plate contained $650 \mu \mathrm{l}$ complete medium with $10 \%$ FBS. After $16 \mathrm{~h}$, the cells were fixed with methanol and stained with $0.1 \%$ crystal violet. Cells in the upper chambers were removed gently and the cells which had invaded into the membranes were imaged under an inverted fluorescent microscope. The number of invasive cells were counted for statistical use. All the aforementioned assays were performed three times.

Western blot analysis. Cells were harvested and lysed in RIPA lysis buffer (Nanjing KeyGen Biotech Co., Ltd., Nanjing, China) supplemented with phenylmethylsulfonyl fluoride (PMSF). Total protein concentration was quantified by BCA assay kit (Beyotime Institute of Biotechnology) and Gen5 Software (CHS 2.06; BioTek Instruments, Winooski, VT, USA). An equal volume of protein samples $(30 \mu \mathrm{g})$ were subjected to $10 \%$ SDS-PAGE and transferred onto polyvinylidene difluoride (PVDF) membranes (EMD Millipore, Billerica, MA, USA). The membranes were then incubated with the primary antibodies, including Snail, $\beta$-catenin, vimentin, cyclin D1, cyclin D3, CDK4, Myc, Cdc42 and GAPDH at $4^{\circ} \mathrm{C}$ overnight and subsequently with the secondary antibody (anti-rabbit IgG, HRP-linked antibody; 1:5,000; cat. no. 7054; Cell Signaling Technology, Danvers, MA, USA) for $1 \mathrm{~h}$ at room temperature. The signals from protein were detected by an advanced ECL detection kit. The rabbit monoclonal antibodies used in the present study were purchased from Cell Signaling Technology, including those against human Snail (1:1,000; cat. no. 3879), $\beta$-catenin $(1: 1,000$; cat. no. 8480), vimentin $(1: 1000$; cat. no. 5741), cyclin D1 (1:1,000; cat. no. 2978), cyclin D3 (1:1,000; cat. no. 2936), CDK4 (1:1,000; cat. no. 12790), c-Myc (1:1,000; cat. no. 13987) and GAPDH (1:1000; cat. no. 5174); Cdc42 (1:800; cat. no. 10155-AP-1) was purchased from Proteintech Group Inc. (Wuhan, China).

MicroRNA sequencing and DataSets. A whole genome microRNA sequencing was carried out by Illumina Hiseq 2500 to detect the dysregulated miRNAs in NPC cell C666-1 and immortalized nasopharyngeal epithelial cell NP69. Data in the GEO DataSets (GSE32960 and GSE36682) was involved in the study (https://www.ncbi.nlm.nih.gov/geo/query/acc.cgi). starBase v2.0 (http://www.Incrnablog.com/starbase-v2-0-fordecoding-rna-interaction-networks/), miRWalk 2.0 (http://zmf. umm.uni-heidelberg.de/apps/zmf/mirwalk2/index.html) and microRNA.org (http://microrna.org/) were applied to predict the potential targets of miR-342-3p.

Statistical analysis. All statistical analyses were performed using SPSS software version 20.0 (IBM Corp., Armonk, NY, USA). To assess the differences among categorical variables, one-way analysis of variance (ANOVA)/Student-NewmanKeuls (SNK) test and independent-sample Student's t-test and Spearman rank correlation were the four major methods used to analyze the results of the present study. $\mathrm{P}<0.05$ was considered to indicate a statistically significant difference.

\section{Results}

miR-342-3p is downregulated in NPC cell lines and tissues. To explore the deregulation of miRNAs in human NPC oncogenesis, a whole genome microRNA sequencing using Illumina Hiseq 2500 was performed and its result revealed that the expression level of miR-342-3p was clearly lower in NPC C666-1 cells than in non-tumor NP69 cells (Table I). To ascertain this finding, we determined the expression level of miR-342-3p in four NPC cell lines including C666-1, 6-10B, $5-8 \mathrm{~F}$ and SUNE2 using RT-qPCR assays, with the immortalized cell line NP69 used as the control. We observed that the expression of miR-342-3p was markedly downregulated in all four NPC cell lines compared with NP69 (Fig. 1B). In 

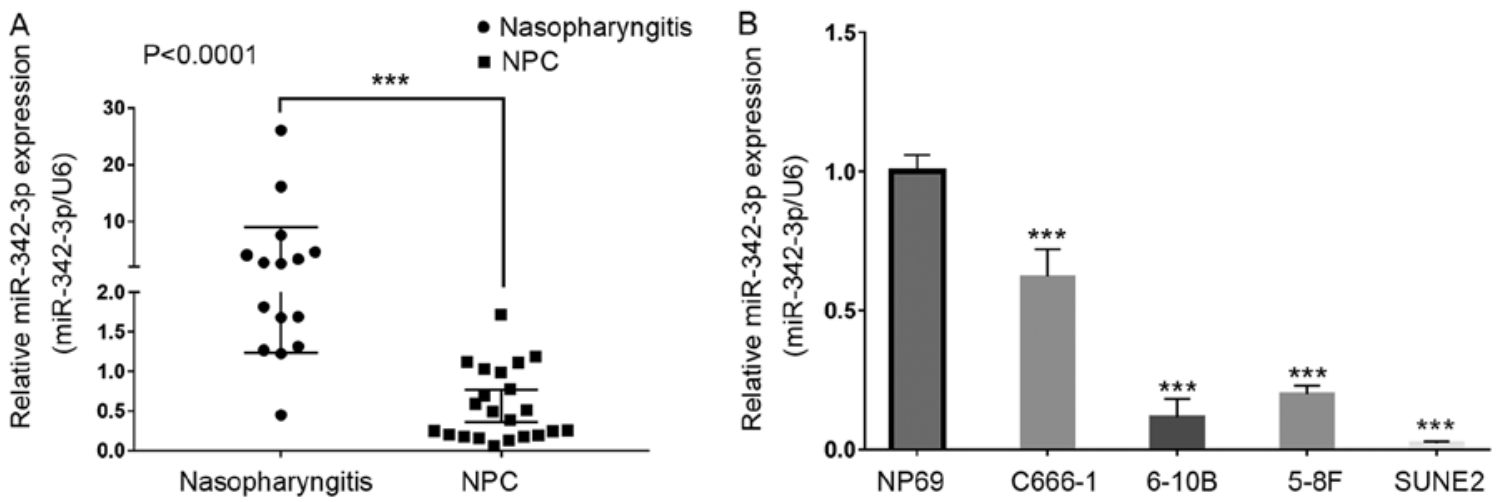

Figure 1. miR-342-3p is downregulated in NPC samples and cell lines. Differences in the expression of miR-342-3p were compared between (A) 22 NPC and 15 nasopharyngitis samples and (B) NPC cell lines and the immortalized nasopharyngeal epithelial cell line NP69. ${ }^{* * *} \mathrm{P}<0.001$. NPC, nasopharyngeal carcinoma.

Table II. The expression of miR-342-3p is reduced in nasopharyngeal carcinoma compared with NP in MicroRNA GEO datasets.

\begin{tabular}{|c|c|c|c|c|c|c|}
\hline \multirow{2}{*}{$\begin{array}{l}\text { MicroRNA GEO datasets } \\
\text { accession no. }\end{array}$} & \multirow[b]{2}{*}{ miRNA_ID } & \multicolumn{2}{|c|}{ No. of cases } & \multirow[b]{2}{*}{$\log 2$ (fold change) } & \multirow[b]{2}{*}{ P-value } & \multirow{2}{*}{$\begin{array}{l}\text { Adjusted } \\
\text { P-value }\end{array}$} \\
\hline & & $\mathrm{NP}$ & NPC & & & \\
\hline GSE32960 & hsa-miR-342-3p & 312 & 18 & -1.479853 (NPC vs. NP) & $7.95 \mathrm{E}-32$ & $6.63 \mathrm{E}-30$ \\
\hline GSE36682 & hsa-miR-342-3p & 62 & 6 & -1.295291 (NPC vs. NP) & 0.0004 & 0.0018 \\
\hline
\end{tabular}

NPC, nasopharyngeal carcinoma; NP, nasopharyngitis.

addition, the expression of miR-342-3p was also detected in 22 NPC and 15 nasopharyngitis samples. Consistent with results in NPC cell lines, it was observed that miR-342-3p was downregulated in NPC compared with nasopharyngitises $(\mathrm{P}<0.0001$, Fig. 1A). Furthermore, data in the GEO DataSets (GSE32960 and GSE36682) revealed that the expression level of miR-342-3p in NPC was significantly lower than that in normal tissues using microarray, which was consistent with our results (Table II).

miR-342-3p inhibits NPC cell proliferation in vitro. To investigate the biological function of miR-342-3p in NPC cells, the miR-342-3p mimics were transfected into 6-10B cells. The overexpression efficiency was validated by RT-qPCR assay (Fig. 2A). miR-342-3p overexpression significantly suppressed NPC cell viability as assessed by CCK-8 assay (Fig. 2B). In addition, compared with the scramble control, colony formation ability of 6-10B cells was also decreased, which was induced by miR-342-3p mimics (Fig. 2C). These findings indicated that miR-342-3p may act as a suppressor in NPC. In addition, cell cycle-related proteins cyclin D1, cyclin D3 and CDK4 were downregulated following miR-342-3p overexpression (Fig. 2D). Our results demonstrated that miR-342-3p suppressed NPC cell proliferation in vitro.

miR-342-3p inhibits NPC cell migration and invasion in vitro. The effects of miR-342-3p overexpression on NPC cell migration and invasion were determined by wound healing and Transwell assays, respectively. In the wound healing assay, the gap filling was markedly decreased in the miR-342-3p-overexpressing 6-10B cells compared with the control cells (Fig. 3A). In the Matrigel-coated Transwell assay, the number of invading cells was markedly decreased in the miR-342-3p-overexpressing 6-10B cells compared with control cells (Fig. 3B). In addition, the expression of transcription factor Snail and mesenchymal markers $\beta$-catenin and vimentin were reduced in the miR-342-3p-overexpressing 6-10B cells (Fig. 3C). Collectively, our results indicated that miR-342-3p inhibited NPC cell migration and invasion.

miR-342-3p directly targets Cdc42 in NPC. To further explore how $\mathrm{miR}-342-3 \mathrm{p}$ regulated proliferation and migration of NPC cells, we used multiple target algorithms (starBase v2.0, miRWalk 2.0 and microRNA.org) to predict candidate targets of miR-342-3p and observed that the 3'UTR of Cdc42 matched the 'seed sequence' of miR-342-3p (Fig. 4A). Luciferase reporter assay revealed that miR-342-3p overexpression inhibited the luciferase activity of wild-type (wt) Cdc42 3'UTR but not the mutant (mt) Cdc42 3'UTR ( $\mathrm{P}<0.01$, Fig. 4B). RT-qPCR and western blot assays confirmed that both the mRNA and protein levels of $\mathrm{Cdc} 42$ were downregulated when miR-342-3p was overexpressed in 6-10B cells (Fig. 4C and D). Our results determined that $\mathrm{Cdc} 42$ was one of the direct targets of miR-342-3p. To further confirm the relationship between miR-342-3p and Cdc42, we analyzed the miR-342-3p expression by RT-qPCR and $\mathrm{Cdc} 42$ protein expression by immunohistochemistry assay simultaneously in 10 NPC samples (Fig. 5A). As displayed in Fig. 4E, miR-342-3p expression was inversely correlated with Cdc42 expression (Fig. 4E 

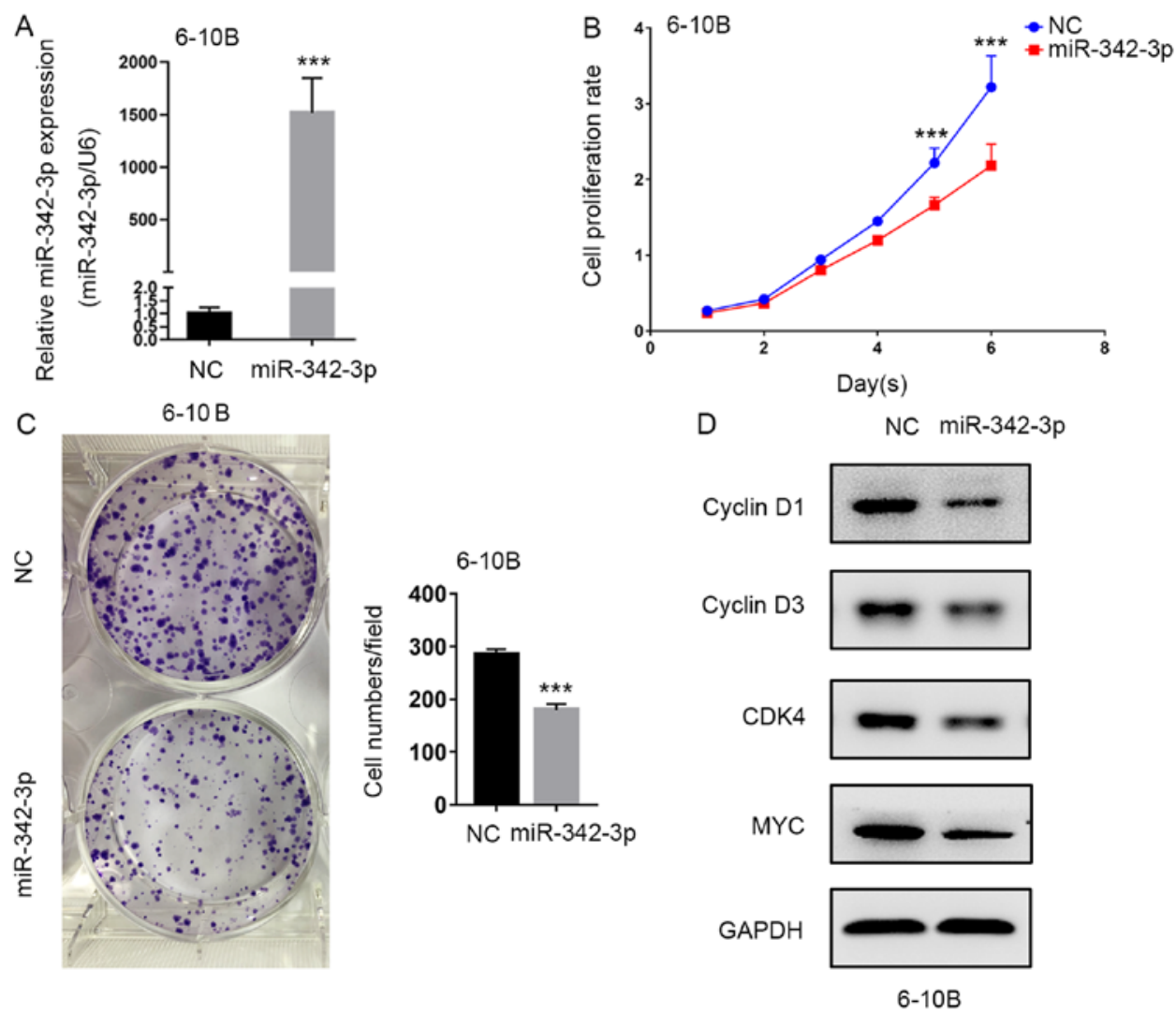

Figure 2. High expression of miR-342-3p inhibits NPC cell growth in vitro. (A) RT-qPCR validation of miR-342-3p overexpression efficiency in 6-10B cells treated with miR-342-3p mimics. (B) A CCK-8 assay revealed that miR-342-3p overexpression in NPC cells delayed cell growth in 6-10B cells. (C) The effects of overexpression of miR-342-3p on the clonogenic ability of 6-10B cells. (D) miR-342-3p-overexpressing 6-10B cells underwent western blot analysis using antibodies specific to cyclin D1, cyclin D3, CDK4, MYC and GAPDH. miR-342-3p mimics markedly inhibited the expression of cyclin D1, cyclin D3, CDK4 and MYC. ${ }^{* * * *} \mathrm{P}<0.001$ vs. NC. NPC, nasopharyngeal carcinoma.

A
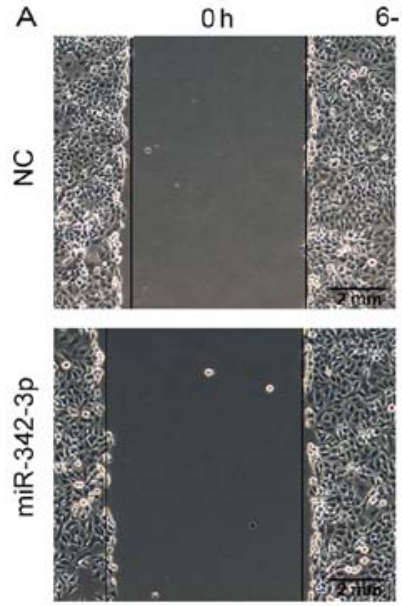

B

NC

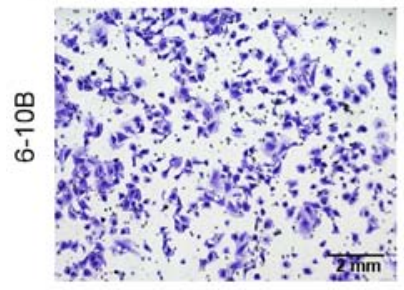

$6-10 B$
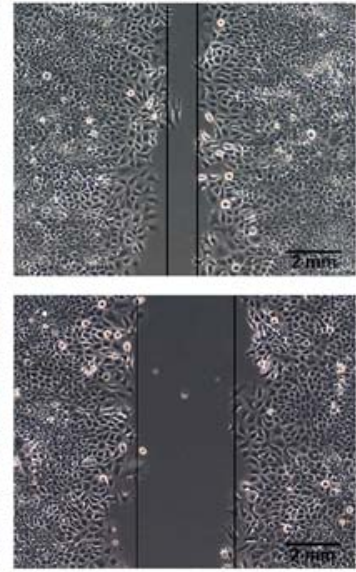

miR-342-3p

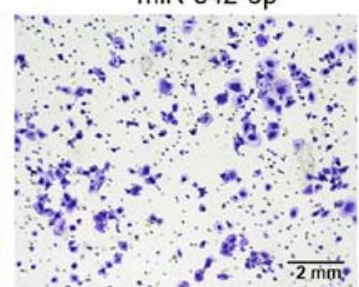

C
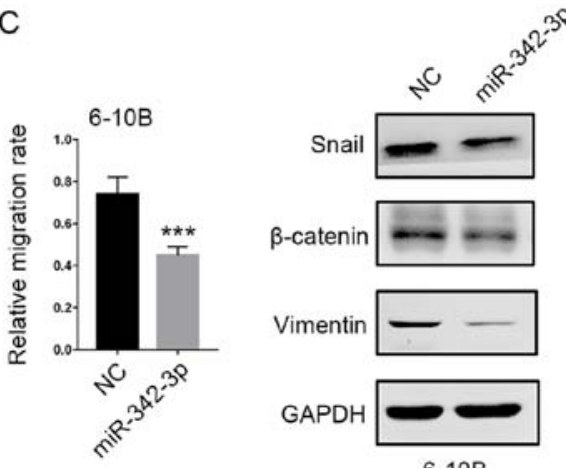

$\beta$-catenin $\cdots$

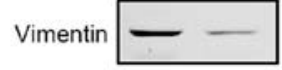

GAPDH

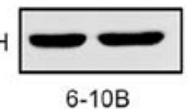

$6-10 B$

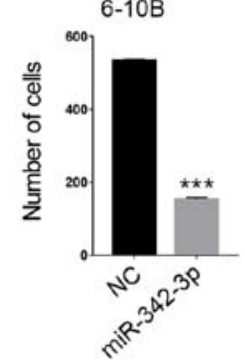

Figure 3. miR-342-3p regulates the migration and invasiveness of NPC cells in vitro. (A) A wound healing assay demonstrated that miR-342-3p overexpression markedly delayed the migration of 6-10B cells. (B) A Transwell assay demonstrated that miR-342-3p overexpression markedly attenuated the invasion of 6-10B cells. ${ }^{* * *} \mathrm{P}<0.001$ vs. $\mathrm{NC}(\mathrm{C})$ A western blotting assay revealed reduced levels of $\beta$-catenin, vimentin and the EMT regulatory factor Snail in 6-10B cells treated with miR-342-3p mimics compared with NC cells. NPC, nasopharyngeal carcinoma; EMT, epithelial-mesenchymal transition. 
A

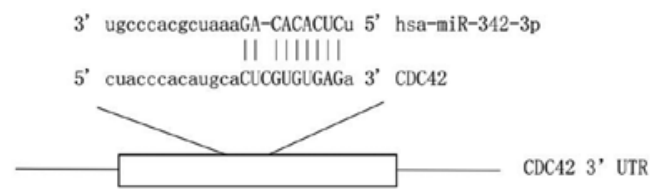

B

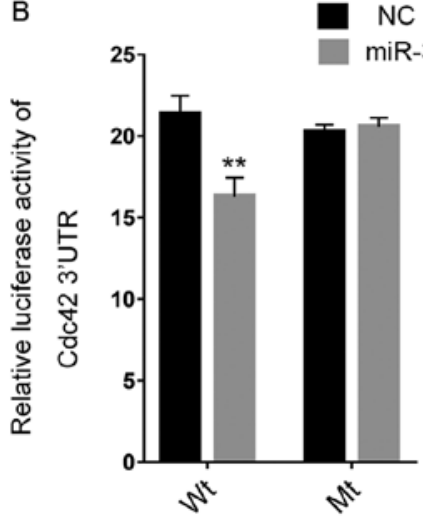

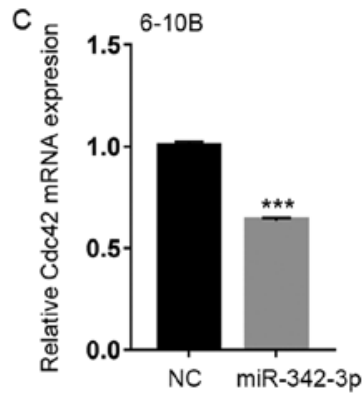

D

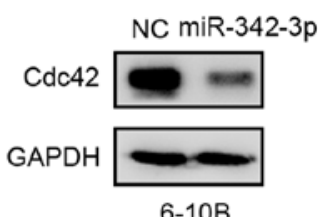

$6-10 B$

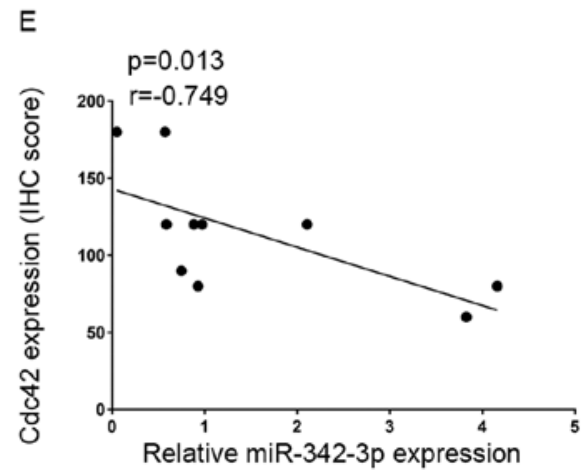

Figure 4. Cdc42 is a direct target of miR-342-3p in NPC. (A) Schematic of predicted miR-342-3p-binding sites in the Cdc42 3'UTR. (B) Luciferase reporter assays in 6-10B cells co-transfected with either the wild-type Cdc42 3'UTR or mutated 3'UTR construct with miR-342-3p or NC oligonucleotides. (C) RT-qPCR analysis of Cdc42 mRNA levels in the 6-10B cells transfected with miR-342-3p mimics or NC oligos. (D) Representative western blot images of Cdc42 in 6-10B cells transfected with NC or miR-342-3p mimics harvested at $72 \mathrm{~h}$. (E) The expression levels of miR-342-3p and Cdc42 were detected simultaneously in 10 NPC samples. Spearman's rank correlation was calculated on the basis of the relative expression levels of miR-342-3p by RT-qPCR and relative protein expression levels (IHC scores) of Cdc $42 .{ }^{* *} \mathrm{P}<0.01,{ }^{* * *} \mathrm{P}<0.001$ vs. NC. NPC, nasopharyngeal carcinoma; IHC, immunohistochemistry.

A

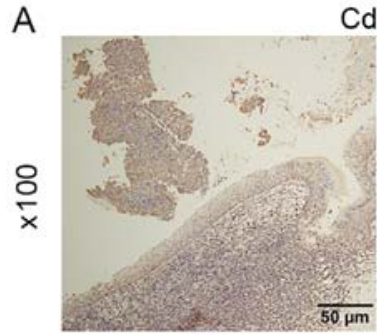

NPC
$\mathrm{Cdc4} 2$

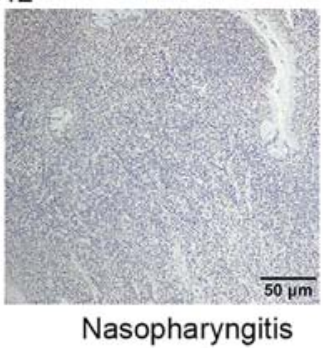

B

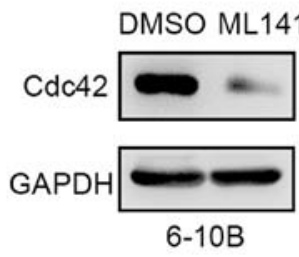

C

DMSO

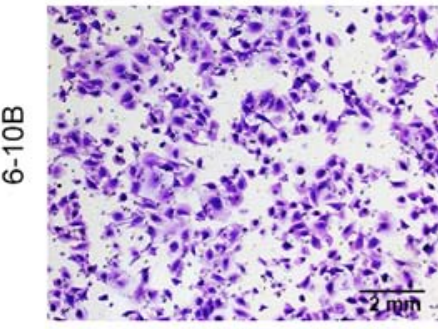

$\mathrm{ML} 141(20 \mu \mathrm{M})$

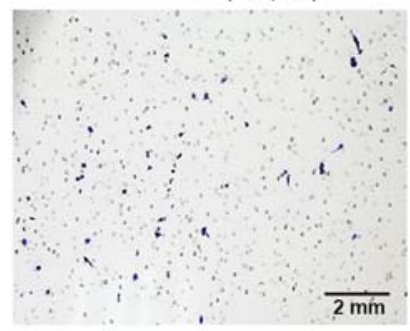

$6-10 B$

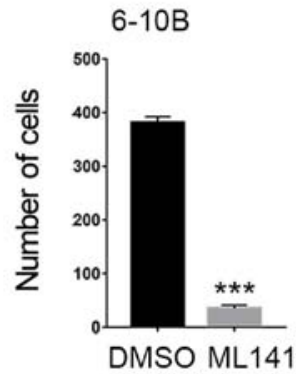

Figure 5. Cdc42 is upregulated in NPC and promotes cell invasiveness. (A) Representative immunohistochemical staining images of Cdc42 in NPC tissues and nasopharyngitis tissues. The staining density of $\mathrm{Cdc} 42$ was higher in tumor cells than in adjacent non-tumor epithelial cells. (B) Western blot analysis revealed that ML141 inhibited Cdc42. (C) A Transwell assay demonstrated that cell invasiveness was markedly reduced in 6-10B cells following treatment with Cdc42 inhibitor ML141 $(20 \mu \mathrm{M}){ }^{* * *} \mathrm{P}<0.001$ vs. DMSO. NPC, nasopharyngeal carcinoma; DMSO, dimethyl sulfoxide.

Spearman's rank correlation coefficient $r=-0.749, \mathrm{P}=0.013$ ) in the same NPC specimens, which indicated that miR-342-3p was actually involved in the in vivo regulation of $\mathrm{Cdc} 42$ in NPC patients.

It has been reported that overexpression of $\mathrm{Cdc} 42$ was detected in different types of human cancer and correlated with increased cancer progression and poorer outcome (16). However, the expression profile and exact role of Cdc42 in NPC were not clear. In the present study, we observed that the expression of $\mathrm{Cdc} 42$ in NPC cells was higher than adjacent non-tumor epithelium as determined by immunohistochemistry (Fig. 5A). To explore the role of Cdc 42 expression in NPC 
cells, we treated 6-10B cells with Cdc42 inhibitor ML141, and observed that ML141 treatment significantly reduced NPC cell invasion ability (Fig. 5B and C). Our findings indicated that miR-342-3p may act as a tumor suppressor in NPC by inhibiting Cdc42.

\section{Discussion}

miR-342 is encoded in an intron of the gene EVL, whose protein product is an actin-associated protein involved in a variety of processes related to cytoskeleton remodeling, cell motility and polarity (17). A previous study reported that both EVL and miR-342 genes were coordinately downregulated in the majority of colorectal cancers due to $\mathrm{CpG}$ island methylation upstream of the EVL/hsa-miR-342 locus (18).

Reduced expression of miR-342 has been demonstrated in colorectal cancer, hepatocellular carcinoma, cervical cancer, osteosarcoma and extranodal natural killer (NK)/T-cell lymphoma, nasal type (ENKTCL) tissues compared with normal tissues (19-22). In addition, research has revealed that miR-342-3p may be used as a potential therapeutic and prognostic biomarker. Breast cancer patients with high miR-342 expression level have significantly better survival compared to patients with low expression (9). Cittelly et al (23) reported that miR-342 was downregulated in tamoxifen resistant breast tumor cell lines and tamoxifen refractory human breast tumors. The expression level of miR-342-3p was reduced in metastatic non-small cell lung cancer samples compared to those in primary tumor samples. Functional studies revealed that miR-342-3p exerted a tumor suppressive role that was operated through regulation of cell proliferation, apoptosis and cell cycle progression, invasion and migration. AGR2 (24), AEG-1 (21), Ikk-g, TAB2 and TAB3 (19), FOXM1 (20), TIAM1 (22) and DNMT1 (25) have been experimentally confirmed as the direct targets for miR-342.

To date, the expression profile and biological function of miR-342-3p in NPC have not been reported. Based on our miRNA sequencing results and RT-qPCR validation, miR-342-3p was identified to be downregulated in NPC tissues and cell lines compared with normal controls. miR-342-3p overexpression inhibited cell proliferation, epithelial-mesenchymal transition (EMT), migration and invasion in NPC cells. These findings were generally consistent with previous studies that suggested a possible tumor suppressor function of miR-342-3p in other cancers.

In the present study, we revealed for the first time that miR-342-3p may exert its function by specifically targeting Cdc42 (cell division control protein) in NPC. Cdc42, a member of the Rho GTPase family, acts as a molecular switch in multicellular pathways. Activated Cdc 42 mediates a signaling cascade leading to the activation of more than twenty downstream effectors, and then influences a variety of cellular responses such as cell polarity, cytoskeleton remolding, proliferation, migration, cellular transformation and gene expression. Cdc 42 is overexpressed in a number of human cancers, and in some instances, has been correlated with poor prognosis. Despite the prominent association of $\mathrm{Cdc} 42$ activation with tumor development and progression, the regulation and oncogenic role of $\mathrm{Cdc} 42$ in NPC cells remains to be elucidated. Epstein-Barr Virus-encoded LMP1 was reported to interact with FGD4 to activate Cdc42 and thereby promote migration of NPC cells (26). A traditional Chinese medicine, berberine, may inhibit the anti-migration and anti-invasion properties of NPC cells through effective inactivation of $\mathrm{Cdc} 42$ (27). These studies revealed that $\mathrm{Cdc} 42$ may be involved in the progression and metastasis in NPC. In the present study, we provided evidence from the luciferase activity assay and western blot analysis that $\mathrm{Cdc} 42$ was a direct target of miR-342-3p. In addition, the protein expression levels of Cdc42 in most NPC cell lines were higher than that in non-neoplastic cell line NP69 as determined by using western blot analysis. Cdc42 expression in NPC biopsies was also higher than adjacent non-tumor epithelium as revealed by immunohistochemistry. These findings indicated that Cdc42 expression was activated in NPC and downregulation of miR-342-3p in NPC may be the cause of Cdc42 activation. In addition, the inhibition of Cdc42 activity by ML141 caused marked reduction of NPC cell invasion ability, indicating that Cdc42 may play an important role in NPC progression.

Our results revealed that reduced expression of miR-342-3p in human NPC contributed to the enhanced proliferation and invasion of NPC cells by direct targeting of the Cdc 42 pathway. Cdc42-selective small-molecule inhibitors have been used in a variety of cancer models, including colon, breast and skin tumors (28-30). Our study provided evidence that miR-342-3p may be used as a potential biomarker and new therapeutic target for $\mathrm{Cdc} 42$ regulation and NPC progression.

\section{Acknowledgements}

Not applicable.

\section{Funding}

The present study was supported by grants from the National Nature Science Foundation of China (nos. 81772884, 81572466, 81772991 and 81629004).

\section{Availability of data and materials}

The datasets used during the present study are available from the corresponding author upon reasonable request.

\section{Authors' contributions}

SM, HW and XFSZ conceived and designed the experiments. SM, LS and MW wrote the study. RX and MZ collected and prepared the tissues of NPC patients. LS and RX performed the experiments. LS, NW, XZ and SM analyzed and interpreted the data. All authors read and approved the manuscript and agree to be accountable for all aspects of the research in ensuring that the accuracy or integrity of any part of the work are appropriately investigated and resolved.

\section{Ethics approval and consent to participate}

The present study was approved by the Research Ethics Committee of Sun Yat-sen University Cancer Center (SYSUCC; Guangzhou, China), and written informed consent was obtained from all patients involved in this study. 


\section{Patient consent for publication}

Not applicable.

\section{Competing interests}

The authors declare that they have no competing interests.

\section{References}

1. Chua MLK, Wee JTS, Hui EP and Chan ATC: Nasopharyngeal carcinoma. Lancet 387: 1012-1024, 2016.

2. Lee AW, Ma BB, Ng WT and Chan AT: Management of nasopharyngeal carcinoma: Current practice and future perspective. J Clin Oncol 33: 3356-3364, 2015.

3. Macfarlane LA and Murphy PR: MicroRNA: Biogenesis, function and role in cancer. Curr Genomics 11: 537-561, 2010.

4. Wang J, Samuels DC, Zhao S, Xiang Y, Zhao YY and Guo Y: Current research on non-coding ribonucleic acid (RNA) Genes 8: E366, 2017.

5. Peng XH, Huang HR, Lu J, Liu X, Zhao FP, Zhang B, Lin SX, Wang L, Chen HH, Xu X, et al: MiR-124 suppresses tumor growth and metastasis by targeting Foxq1 in nasopharyngeal carcinoma. Mol Cancer 13: 186, 2014

6. Liu Y, Li Z, Wu L, Wang Z, Wang X, Yu Y, Zhao Q and Luo F: MiRNA-125a-5p: A regulator and predictor of gefitinib's effect on nasopharyngeal carcinoma. Cancer Cell Int 14: 24, 2014.

7. Wu RS, Qiu EH, Zhu JJ, Wang JR and Lin HL: MiR-101 promotes nasopharyngeal carcinoma cell apoptosis through inhibiting Ras/Raf/MEK/ERK signaling pathway. Eur Rev Med Pharmaco Sci 22: 150-157, 2018

8. Xia H, Ng SS, Jiang S, Cheung WK, Sze J, Bian XW, Kung HF and Lin MC: miR-200a-mediated downregulation of ZEB2 and CTNNB1 differentially inhibits nasopharyngeal carcinoma cell growth, migration and invasion. Biochem Biophys Res Commun 391: 535-541, 2010.

9. Yang X, Ni W and Lei K: miR-200b suppresses cell growth, migration and invasion by targeting notch1 in nasopharyngeal carcinoma. Cell Physiol Biochem 32: 1288-1298, 2013.

10. Shao Q, Zhang P, Ma Y, Lu Z, Meng J, Li H, Wang X, Chen D, Zhang M, Han Y, et al: MicroRNA-139-5p affects cisplatin sensitivity in human nasopharyngeal carcinoma cells by regulating the epithelial-to-mesenchymal transition. Gene 652: 48-58, 2018

11. Zhao M, Luo R, Liu Y, Gao L, Fu Z, Fu Q, Luo X, Chen Y, Deng X Liang Z, et al: miR-3188 regulates nasopharyngeal carcinoma proliferation and chemosensitivity through a FOXO1-modulated positive feedback loop with mTOR-p-PI3K/AKT-c-JUN. Nat Commun 7: 11309, 2016.

12. Zhang JX, Qian D, Wang FW, Liao DZ, Wei JH, Tong ZT, Fu J, Huang XX, Liao YJ, Deng HX, et al: MicroRNA-29c enhances the sensitivities of human nasopharyngeal carcinoma to cisplatin-based chemotherapy and radiotherapy. Cancer Lett 329: 91-98, 2013.

13. Ma F, Wang Z, Wang J, Liu X and Hu C: MicroRNA-19a promotes nasopharyngeal carcinoma by targeting transforming growth factor $\beta$ receptor 2. Exp Ther Med 14: 1419-1426, 2017.

14. Wu W, Chen X, Yu S, Wang R, Zhao R and Du C: microRNA-222 promotes tumor growth and confers radioresistance in nasopharyngeal carcinoma by targeting PTEN. Mol Med Rep 17: $1305-1310,2018$
15. Livak KJ and Schmittgen TD: Analysis of relative gene expression data using real-time quantitative PCR and the $2^{-\Delta \Delta C_{\mathrm{T}}}$ method. Methods 25: 402-408, 2001.

16. Arias-Romero LE and Chernoff J: Targeting Cdc42 in cancer. Expert Opin Ther Targets 17: 1263-1273, 2013.

17. Krause M, Dent EW, Bear JE, Loureiro JJ and Gertler FB Ena/VASP proteins: Regulators of the actin cytoskeleton and cell migration. Annu Rev Cell Dev Biol 19: 541-564, 2003.

18. Grady WM, Parkin RK, Mitchell PS, Lee JH, Kim YH, Tsuchiya KD, Washington MK, Paraskeva C, Willson JK, $\mathrm{Kaz} \mathrm{AM}$, et al: Epigenetic silencing of the intronic microRNA hsa-miR-342 and its host gene EVL in colorectal cancer. Oncogene 27: 3880-3888, 2008.

19. Zhao L and Zhang Y: miR-342-3p affects hepatocellular carcinoma cell proliferation via regulating $\mathrm{NF}-\mathrm{kB}$ pathway. Biochem Biophys Res Commun 457: 370-377, 2015.

20. Li XR, Chu HJ, Lv T, Wang L, Kong SF and Dai SZ: miR-342-3p suppresses proliferation, migration and invasion by targeting FOXM1 in human cervical cancer. FEBS Lett 588: 3298-3307, 2014.

21. Zhang S, Liu L, Lv Z,LiQ, Gong W and Wu H: MicroRNA-342-3p inhibits the proliferation, migration, and invasion of osteosarcoma cells by targeting astrocyte-elevated gene-1 (AEG-1). Oncol Res 25: 1505-1515, 2017.

22. Huang H, Fan L, Zhan R, Wu S and Niu W: Expression of microRNA-10a, microRNA-342-3p and their predicted target gene TIAM1 in extranodal NK/T-cell lymphoma, nasal type. Oncol Lett 11: 345-351, 2016.

23. Cittelly DM, Das PM, Spoelstra NS, Edgerton SM, Richer JK, Thor AD and Jones FE: Downregulation of miR-342 is associated with tamoxifen resistant breast tumors. Mol Cancer 9: 317, 2010.

24. Xue X, Fei X, Hou W, Zhang Y, Liu L and Hu R: miR-342-3p suppresses cell proliferation and migration by targeting AGR2 in non-small cell lung cancer. Cancer Lett 412: 170-178, 2018.

25. Wang H, Wu J, Meng X, Ying X, Zuo Y, Liu R, Pan Z, Kang T and Huang W: MicroRNA-342 inhibits colorectal cancer cell proliferation and invasion by directly targeting DNA methyltransferase 1. Carcinogenesis 32: 1033-1042, 2011.

26. Liu HP, Chen CC, Wu CC, Huang YC, Liu SC, Liang Y, Chang KP and Chang YS: Epstein-Barr virus-encoded LMP1 interacts with FGD4 to activate $\mathrm{Cdc} 42$ and thereby promote migration of nasopharyngeal carcinoma cells. PLoS Pathog 8: e1002690, 2012.

27. Tsang CM, Lau EP, Di K, Cheung PY, Hau PM, Ching YP, Wong YC, Cheung AL, Wan TS, Tong Y, et al: Berberine inhibits Rho GTPases and cell migration at low doses but induces G2 arrest and apoptosis at high doses in human cancer cells. Int J Mol Med 24: 131-138, 2009.

28. Bradshaw-Pierce EL, Pitts TM, Tan AC, McPhillips K, West M, Gustafson DL, Halsey C, Nguyen L, Lee NV, Kan JL, et al: Tumor P-glycoprotein correlates with efficacy of PF-3758309 in in vitro and in vivo models of colorectal cancer. Front Pharmacol 4: 22, 2013.

29. Pelish HE, Peterson JR, Salvarezza SB, Rodriguez-Boulan E, Chen JL, Stamnes M, Macia E, Feng Y, Shair MD and Kirchhausen T: Secramine inhibits Cdc42-dependent functions in cells and Cdc42 activation in vitro. Nat Chem Biol 2: 39-46, 2006.

30. Murray BW, Guo C, Piraino J, Westwick JK, Zhang C, Lamerdin J, Dagostino E, Knighton D, Loi C, Zager M, et al: Small-molecule p21-activated kinase inhibitor PF-3758309 is a potent inhibitor of oncogenic signaling and tumor growth. Proc Natl Acad Sci USA 107: 9446-9451, 2010. 